\title{
RECURSO DE ENFRENTAMENTO OU MECANISMO DE DEFESA? A FÉ NO LEITO DE HOSPITAL ${ }^{1}$
}

\author{
Marisa Ferreira Rocha - UESPI ${ }^{2}$, Déborah Éllen de Matos Ribeiro - UESPI', \\ Ana Rosa Rebelo Ferreira de Carvalho - UESPI ${ }^{4}$, Cleidiane Araújo Pinheiro - HGV ${ }^{5}$
}

\section{RESUMO}

O presente artigo trata-se de um relato de experiência de um estágio supervisionado em Psicologia Hospitalar realizado em um hospital público de alta complexidade, que objetivou a vivência profissional na assistência psicológica que envolvem os processos saúde-doençacuidado. Destaca-se, a partir desta experiência, a observação da emergência da fé nos discursos dos pacientes como forma de enfrentar o processo de adoecimento e hospitalização, a partir disso, procurou-se refletir sobre a influência desta estratégia de enfrentamento nos processos supracitados. Este estudo tem o propósito de integrar a prática das intervenções psicológicas vivenciadas ao estudo da literatura científica inerente à psicologia hospitalar. Os métodos utilizados foram observação participante, no qual foi possível identificar as repetições de cada paciente sobre a fé, e levantamento bibliográfico para evidenciar as perspectivas de como age aquela no processo de hospitalização. A literatura aponta que o mesmo recurso pode adquirir caráter tanto positivo como negativo para o processo de adoecimento, e somente se compreende se avaliado suas utilizações nos discursos, gestos, e contexto do paciente.

Palavras-chave: Processo de adoecimento. Coping. Religiosidade/espiritualidade.

\begin{abstract}
This article is an experience report of a supervised internship in Hospital Psychology conducted in a public hospital of high complexity, which aimed at professional experience in psychological care involving health-disease-care processes. Based on this experience, we highlight the observation of the emergence of faith in patients' discourses as a way to face the process of illness and hospitalization; from this, we sought to reflect on the influence of this coping strategy on the above mentioned processes. This study aims to integrate the practice of psychological interventions experienced in the study of the scientific literature inherent to hospital psychology. The methods used were participant observation, in which it was possible to identify the repetitions of each patient's faith, and bibliographic survey to highlight the perspectives of
\end{abstract}

\footnotetext{
${ }^{1}$ Trabalho apresentado no Congresso Brasileiro Ciência e Sociedade (CBCS 2019), promovido pelo Centro Universitário Santo Agostinho, de 03 a 05 de outubro de 2019, em Teresina-PI.

2 Psicóloga pela Universidade Estadual do Piauí-UESPI: marisarocha.psi@gmail.com

${ }^{3}$ Psicóloga pela Universidade Estadual do Piauí-UESPI: deborahellenmr@ hotmail.com

${ }^{4}$ Mestre em Psicologia, Docente do curso de Psicologia da Universidade Estadual do Piauí-UESPI: anarosa.carvalho@bol.com.br

${ }^{5}$ Especialista em Qualidade e Segurança no Cuidado ao Paciente, Psicóloga do Hospital Getúlio Vargas PI: cleidianeapinheiro@gmail.com
} 


\section{cONGQESSOC CIENCIASSOCIEDADE \\ Inovação, Diversidaale e Sustentahilitilade}

ANAIS CBCS 2019 | 3 a 5 de outubro de 2019 | Centro Universitário Santo Agostinho - Teresina - P|

how the patient acts in the hospitalization process. The literature points out that the same resource can acquire both a positive and negative character for the process of illness, and can only be understood if its uses are evaluated in the patient's discourses, gestures, and context.

Keywords: Disease process. Coping. Religiosity/spirituality.

\section{INTRODUÇÃO}

A prática aqui descrita resultou da vivência de Estágio Supervisionado em Psicologia hospitalar de uma universidade pública na cidade de Teresina, Piauí. O estágio desenvolveu-se em um hospital público, referência no estado por ser realizar procedimentos de alta complexidade. A partir desta experiência, do contato com os pacientes, acompanhantes e equipe, foi observada uma forte referência à espiritualidade, o que motivou a busca para o seguinte questionamento: a fé no leito de hospital pode ser caracterizada como recurso de enfrentamento ou mecanismo de defesa?

Neste artigo busca-se discutir a presença da fé e o papel que ela desempenha durante a hospitalização. E para isso, tem-se como objetivos secundários estabelecer definições claras do que distingue mecanismo de defesa de recurso de enfrentamento, de acordo com a psicologia; avaliar a presença de fé nos relatos dos pacientes; e, refletir sobre o espaço e as funções que ocupa a fé no processo saúde-doença.

O processo de adoecimento acontece de forma inesperada na vida de um indivíduo, altera seu cotidiano, traz consigo reflexões acerca da finitude da vida, coloca em cena as relações que um indivíduo constitui e pode levantar uma série de demandas que podem influir no progresso de um possível tratamento. Caracteriza-se por um período de crise em que há um desequilíbrio que não pode ser adiado diante de uma ameaça à vida e tendo o sujeito que se mobilizar para encontrar estratégias de enfrentamento, essa vivência acontece de forma diferente em cada pessoa.

A crise geralmente vem acompanhada de ansiedade, medo ou depressão, e o processo de enfrentamento ou coping pode ser descrito como o conjunto de estratégias utilizadas por pessoas para se adaptarem a circunstâncias adversas ou estressantes que 


\section{CONGEESSOC CIENCIAESOCIEDADE

ANAIS CBCS 2019 | 3 a 5 de outubro de 2019 | Centro Universitário Santo Agostinho - Teresina - PI

sobrecarregam o excedem os recursos pessoais. Adoecimento e hospitalização são uma situação de crise, considerando que por si só causam ansiedade e stress por ser num ambiente marcado por insegurança, perda da independência, perda do poder de decisão, com uma dinâmica diferente da habituada e que há necessidade de adaptação (ANTONIAZZI, DELL'ÁGLIO E BANDEIRA, 1998; SEVERO, GIRARDON-PERLINI, 2005).

As estratégias de enfrentamento são processos de controle utilizados para administrar as a relação entre as demandas estressantes requeridas pela doença e as respostas que o indivíduo produz perante as mesmas. $O$ enfrentamento pode ser focalizado no problema, quando o indivíduo busca remover ou abrandar a fonte estressora. Ou focalizado na emoção, quando não atua na situação origem do problema, mas negocia com as emoções, modificando o significado da situação, não importando se isso é feito de forma realista ou com distorção da realidade. Objetiva regular o estado emocional associado ao estresse, para manter uma autoestima positiva, esperança e bem-estar (COSTA E LEITE, 2009).

Os recursos que cada pessoa vai utilizar dependem de características pessoais como, crenças ideológicas, experiências prévias de coping, forma habitual de lidar com stress, saúde física, variáveis psicológicas e dos recursos disponíveis no contexto, como família e rede de suporte social (ANTONIAZZI, DELL'ÁGLIO E BANDEIRA, 1998). Em decorrência da expansão do modelo biopsicossocial adotado pela OMS em um conceito ampliado de saúde, a religião e espiritualidade têm sido reconhecidas por sua relevância nas abordagens ao tratamento.

O coping religioso/espiritual (CRE) é uma estratégia muito presente nos hospitais, definida como o uso da fé e comportamento religioso para lidar com o estresse advindo de crises, prevenindo ou aliviando as consequências emocionais negativa, se relacionando com melhores índices de qualidade de vida e saúde física e mental (PANZINI E BANDEIRA, 2007).

Benites, Neme e Santos (2017), destacam a importância de aspectos ligados à espiritualidade no processo de cura e reabilitação de doenças, apontando estudos que 


\section{CONGEESSOC CIENCIAESOCIEDADE

ANAIS CBCS 2019 | 3 a 5 de outubro de 2019 | Centro Universitário Santo Agostinho - Teresina - P|

demonstram a existência de relações entre religiosidade/espiritualidade à qualidade de vida, o que vem dando maior atenção à dimensão espiritual do sujeito em cuidados de saúde.

Os autores trazem a distinção de religiosidade como um conjunto de crenças, linguagem e práticas institucionalizadas que produzem explicações acerca da vida e da morte; e espiritualidade como algo universal sem restrição à religião, cultura ou grupo, mas que envolve valores pessoais e íntimos com relação ao que dá sentido à vida, esperança/vontade para viver e de ter fé em si mesmo, nos outros ou Deus.

Em revisão da literatura da área, Gobatto e Araujo (2010) evidenciam uma crescente preocupação em se compreender e avaliar o CRE em circunstâncias potencialmente estressantes como são as internações hospitalares. Concluem que é fundamental que o psicólogo atuante nesse contexto considere as estratégias positivas do coping religioso/espiritual, e utilize-o como forma de buscar maior adesão ao tratamento, facilitar o acesso a redes de suporte e integração social, produzir sentidos à vida e reduzir os sintomas depressivos.

Stroppa e Moreira-Almeida (2008), afirmam que há uma tradição ocidental de ênfase numa relação pessoal com Deus e com o próximo, especialmente quando se enfrenta situações difíceis como o adoecimento. Essa percepção de relação com Deus, quando positiva, pode ter importantes consequências, proporcionando ao enfermo, maior aceitação, autoconfiança, firmeza e adaptação às situações difíceis, reduzindo a sensação de desamparo e perda do controle, que acompanham doenças físicas.

Ao passo que o coping religioso/espiritual pode refletir consequências prejudiciais ao indivíduo, quando por exemplo o indivíduo passa a:

questionar a existência, amor ou atos de Deus, delegar a Deus a resolução dos problemas, sentir insatisfação ou descontentamento em relação a Deus ou frequentadores e membros de instituição religiosa, redefinir o estressor como punição divina ou forças do mal, trazendo como consequência índices mais baixos de qualidade de vida. (SCHLEDER, PAREJO, PUGGINA, SILVA, 2013). 


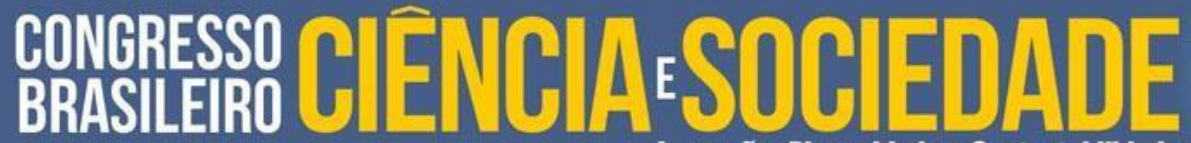 \\ Inovação, Diversidaale e Sustentahilitilatle}

ANAIS CBCS 2019 | 3 a 5 de outubro de 2019 | Centro Universitário Santo Agostinho - Teresina - PI

Assim, frente ao adoecimento e suas repercussões psicossociais podemos observar a importância da espiritualidade na forma como é vivenciado o adoecimento e qual o sentido se dá para a vida a partir daquele momento. Destacamos aqui a espiritualidade como uma forte estratégia de enfrentamento e questiona-se se sua manifestação também pode se caracterizar como um mecanismo de defesa, uma forma de se "esquivar" das responsabilidades exigidas pelo processo saúde-doença.

\section{METODOLOGIA}

O presente artigo refere-se a um relato de experiência de estágio supervisionado no ano de 2017, efetuado pelas discentes do curso de Psicologia da Universidade Estadual do Piauí-UESPI. O Estágio foi realizado no Hospital de Getúlio Vargas na cidade de Teresina-PI, um dos maiores e mais bem equipados do estado, que se diferencia por realizar procedimentos de alta complexidade em várias especialidades médicas, via SUS (Sistema Único de Saúde), além de contar com programas de Residência Médica e Multiprofissional.

Os tratamentos oferecidos abrangem diversas áreas, totalizando 15 clínicas na unidade de internação: Médica, Cirúrgica, Ortopédica, Ginecológica, Neurológica, Nefrológica e Hemodiálise, Otorrinolaringológica, Pneumológica, Dermatológica, Urológica, Oftalmológica, Cardiológica e Vascular. Dispondo ainda, de duas Unidades de Terapia Intensiva (UTI) e dos serviços ambulatoriais.

A referida instituição possui o Serviço de Psicologia Hospitalar composto por duas profissionais que realizam atendimentos, uma na clínica Neurológica e UTI, e outra a partir de demandas solicitadas nas demais clínicas. Existem ainda em funcionamento paralelo, um profissional responsável pela clínica Nefrológica e Hemodiálise e o Serviço de Psicologia Clínica em ambulatório composto por outros profissionais.

O Estágio possuiu carga horária total de $100 \mathrm{~h}$, divididas em atividades práticas de atendimentos nas unidades de internação e de supervisão. Esta última, concebida como momento importante para socialização do conhecimento através de trocas de 


\section{CONGEESSOC CIENCIAESOCIEDADE

ANAIS CBCS 2019 | 3 a 5 de outubro de 2019 | Centro Universitário Santo Agostinho - Teresina - P|

experiência, esclarecimento de dúvidas e orientação, foi conduzida pela professora da disciplina e pela profissional de psicologia da instituição campo de práticas.

Já as atividades práticas, consistiram em planejando e executando ações que visando a prevenção de problemas psicológicos relacionados ao adoecimento e processo de hospitalização, promoção, avaliação, reabilitação da saúde física e psicológica. Atua em diferentes níveis de tratamento, acompanhando pacientes e seus familiares em seus anseios, expectativas e angústias.

Desta forma, o processo metodológico utilizado foi o da observação participante, no qual o pesquisador vivencia pessoalmente o evento na sua situação natural, para melhor entendê-lo, percebendo e agindo diligentemente de acordo com as suas interpretações daquele mundo, participa nas relações sociais e procura desenvolver um entendimento científico da situação observada (MAY, 2001).

Mediante observação feita durante os atendimentos e relatos em supervisão, destacou-se a presença constante da temática espiritualidade trazida pelos pacientes, diante disso a indagou-se sobre o papel da religiosidade no processo de hospitalização.

\section{RESULTADOS E DISCUSSÃO}

A partir dos tópicos levantados e da experiência advinda do campo de estágio aqui explanado, verifica-se, portanto, forte presença da "fé" no espaço hospitalar. O hospital em questão além de possuir capela própria, conta com bancos e mesa em um pátio central onde são intercaladas missas católicas e cultos evangélicos em diferentes períodos do dia afim de reunir equipe, acompanhantes e pacientes que se interessem em participar.

Por vezes observou-se convites feitos por parte da equipe de funcionários aos acompanhantes e também aos estagiários. Em certa oportunidade também pode-se observar a distribuição de exemplares de bíblias nos corredores da instituição em conjunto com a visita aos leitos por grupos vinculados a igrejas.

No que diz respeito a postura da equipe com que se teve contato direto nas

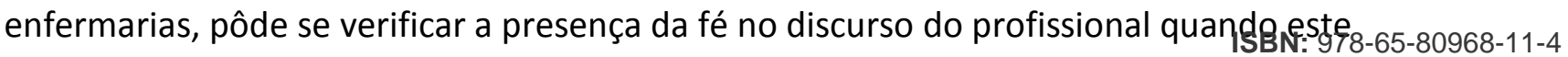




\section{CONGQEESSOCIENCIA:SOCIEDADE

ANAIS CBCS 2019 | 3 a 5 de outubro de 2019 | Centro Universitário Santo Agostinho - Teresina - P a trazia como estratégia de vinculação ao paciente. Percebe-se aqui, a utilização do coping espiritual/religioso como estratégia positiva, como recomendam Gobatto e Araujo (2010). Porém para isso, o profissional precisa conhecer a existência de crenças do paciente e que espaço a espiritualidade/religiosidade tem em sua vida, para não incorrer em doutrinação. No entanto não houveram contatos suficientes para afirmar até que ponto tal espiritualidade influi na prática desses profissionais.

Quanto aos atendimentos realizados aos pacientes e acompanhantes, em sua grande maioria trouxeram algum conteúdo religioso ao expressar expectativas perante o prognóstico e/ou conteúdos relacionados ao morrer. Os discursos manifestaram-se em forma de preces: "peço que Deus me tire dessa situação" "oro muito a Deus todos os dias"; aceitação da condição, em que é complexo identificar até que ponto o paciente se responsabiliza na busca da "cura", ou delega a outrem a resolução dos problemas, como em "seja feita a Tua vontade" .

Os mecanismos também foram manifestados por meio de gestos, ou acessórios religiosos como o terço e a bíblia. Tal como certa paciente, que apresentou angústia por ter esquecido os óculos de grau em casa, ficando impossibilitada de fazer a leitura diária da bíblia que carregava consigo. O processo de hospitalização é permeado por diversas nuances, relações se reconfiguram e são expressadas no leito de formas singulares.

Ao contrário do que imaginado, distinguir se a fé verbalizada é para um sujeito uma estratégia de enfrentamento ou um recurso para não lidar diretamente com o adoecer, não é tão simples. É imprescindível avaliar cada contexto para que se compreenda a fé como uma ferramenta que pode auxiliar no acompanhamento de um dado paciente, ou ainda avaliar até que ponto ela pode esconder algo que está por emergir, e se esse emergir é o ideal naquele momento, se há condições suficientes para se lidar com isso.

\section{CONSIDERAÇÕES FINAIS}

Depreende-se desta experiência, uma percepção de Psicologia Hospitalar como uma prática que objetiva construção de um espaço de atenção integral ao paciente que sofre, possibilitando perceber todos os sentimentos gerados e causados pela ISBN: 978-65-80968-11-4 


\section{CONGEESSOC CIENCIAESOCIEDADE \\ Inovação, Diversidaale e Sustentahilitilade}

ANAIS CBCS 2019 | 3 a 5 de outubro de 2019 | Centro Universitário Santo Agostinho - Teresina - P|

hospitalização, e favorecer um amplo suporte na esfera biopsicossocial ao paciente e sua família.

Foi possível perceber ainda, que a dimensão espiritual/religiosa influencia no modo como pessoas lidam com situações. Em especial quando se precisa lidar com situações adversas da vida, esta ocupa um lugar de destaque na vida das pessoas sendo tão fundamental quanto são as outras formas de enfrentamento.

Desta forma, é necessário que os profissionais conheçam sobre o CRE, a fim de utiliza-lo como recurso no planejamento do cuidado, é também imprescindível conhecer a espiritualidade dos pacientes, a fim de que forneçam uma assistência adequada. Para compreender os discursos emergidos, faz-se necessário entender sobre religião e espiritualidade e como podemos nos instrumentalizar com esses temas, para interpretar e ver surgir produções de sentidos.

Ainda se fazem necessárias mais pesquisas sobre a temática, tendo em vista a complexidade que tal dimensão pode tomar na vivência da hospitalização, em um primeiro momento pode-se relatar que a experiência de avaliar conteúdos ligados à espiritualidade facilitou encorajar a reflexão sobre questões ligadas à vida, se configurando como uma possibilidade de intervenção no âmbito da psicologia hospitalar.

\section{REFERÊNCIAS}

Antoniazzi A.S, Dell'Aglio D.D, Bandeira DR. [The concept of coping: a theoretical review]. Estudos de Psicologia, Natal, 3(2), p. 273-94, 1998.

Benites, A.C., Neme, CM.B., \& Santos, M.A. Significados da espiritualidade para pacientes com câncer em cuidados paliativos. Estudos de Psicologia. Campinas, 34(2), 269-279, 2017.

Costa, P.; Leite R. C. B. O. Estratégias de enfrentamento utilizadas pelos pacientes oncológicos submetidos a cirurgia mutiladora. Revista Brasileira de Cancerologia. 55(4), p. 355- 64, 2009. 


\section{congGESSOCOENCIAESOCIEDADE}

ANAIS CBCS 2019 | 3 a 5 de outubro de 2019 | Centro Universitário Santo Agostinho - Teresina - P|

Gobatto, C.A., \& Araujo, T.C.C.F. Coping religioso-espiritual: reflexões e perspectivas para a atuação do psicólogo em oncologia. Revista da SBPH. 13(1),p. 52-63, 2010.

MAY, T. Pesquisa social. Questões, métodos e processos. Porto Alegre, Artmed, 2001.

PANZINI, Raquel Gehrke; BANDEIRA, Denise Ruschel. Coping (enfrentamento) religioso / espiritual. Revista Psiquiatria Clínica, São Paulo, v. 34, supl. 1, p. 126-135, 2007.

Severo, G. C.; Girardon-Perlini, N. M. O. Estar internado em Unidade de Terapia Intensiva: percepção de pacientes. Scientia Medica, Porto Alegre: PUCRS, v. 15, n. 1, jan./mar, 2005.

Schleder, Letícia Preti; Parejo, Lucineia Stach; Puggina, Ana Cláudia; Silva, Maria Júlia Paes da. Espiritualidade dos familiares de pacientes internados em unidade de terapia intensiva. Acta Paul Enferm. 26(1), p.71-8, 2013.

Stroppa, A; Moreira-Almeida, A. Religiosidade e saúde. Saúde e Espiritualidade: uma nova visão da medicina. Belo Horizonte: Inede, p. 427- 443, 2008. 